\title{
Mathematical Model for Cluster-Inclusion's Collision-Growth in Inclusion Cloud at Continuous Casting Mold
}

\author{
Hong $\mathrm{LEI}^{1{ }^{1 *}}{ }^{1}$ Yan $\mathrm{ZHAO}^{21}$ and Dian-Qiao GENG ${ }^{1)}$ \\ 1) Key Laboratory of Electromagnetic Processing of Materials, Ministry of Education, Northeastern University, Shenyang, \\ Liaoning Province, 110819 P.R. China. \\ 2) School of Materials and Metallurgy, Northeastern University, Shenyang,
}

Liaoning Province, 110819 P.R. China.

(Received on January 28, 2014; accepted on March 5, 2014)

\begin{abstract}
The inclusion's morphology and size play a strong role in the steel product quality, so it is very important to have a deep insight into the inclusion's collision-growth in the continuous caster. In order to describe the formation of the cluster-inclusion, a mathematical model and the related source code are developed to trace the inclusion's movement and collision-growth in the inclusion cloud. Such a model includes two sub-models. Firstly, the spatial distribution of turbulent flow and the inclusion's number density and the characteristic radius after Stokes collision and turbulent collision are obtained by Eulerian approach. Secondly, the inclusion's trajectory and the related inclusion fractal growth are predicted by Lagrangian approach. Numerical results show that the spatial distributions of the inclusion volumetric concentration, number density and characteristic radius have some features of the upper and lower recirculation zone due to the fluid flow. For an inclusion particle, the bigger inclusion has more chances to catch other inclusions and forms a more complex cluster-structure. Among the forces acting on the inclusion, the pressure gradient force, Basset history force, the visual mass force, the gravitational force and the buoyancy force should be considered in order to describe the inclusion's exact motion. Furthermore, the pressure gradient force, the Basset force, the visual mass force follow the same variation rule along with the inclusion's trajectory.
\end{abstract}

KEY WORDS: continuous casting mold; cluster inclusion; mass-population conservation model; Stokes collision; turbulent collision; random rain model; Monte-Carlo method; Euler-Lagrange approach.

\section{Introduction}

As the last metallurgical reactor having molten steel, the continuous casting mold is a vital element of steelmaking process because it is the last opportunity to remove the inclusions from the molten steel. In spite of long-standing interest and research effort, the problem about the collisiongrowth among inclusions is far from being fully resolved because there is complex mutual interaction between the inclusion and the molten steel.

Fundamental points to understand the characteristics of inclusion movement, collision and aggregation in molten steel should be based on the unsteady inclusion-moving process. Theories about inclusion movement in the simplified conditions such as steady fluid flow are well established, ${ }^{1-3)}$ and collision and aggregation among inclusions also can be modeled in spite of the variety and complexity of its aspects, ${ }^{4-6)}$ but the theory for realistic situations of inclusion behavior in the molten steel is still lacking. These complex phenomena will become understood only when the interactive coupling of fluid dynamics and inclusion dynamics are clarified. ${ }^{7,8)}$

* Corresponding author: E-mail: leihong@epm.neu.edu.cn DOI: http://dx.doi.org/10.2355/isijinternational.54.1629
With respect to the inclusion movement and collisiongrowth, most researches are based on Eulerian approach or Lagrangian approach. Both of the approaches have their own advantage and disadvantage.

For Eulerian approach, ${ }^{1,9,10)}$ the governing equation for inclusion transport, which is written in the general form of Eq. (1), can be solved by the finite volume method.

$$
\frac{\partial\left(\rho \overline{\mathrm{u}}_{\mathrm{i}} \Phi\right)}{\partial \mathrm{x}_{\mathrm{i}}}=\frac{\partial}{\partial \mathrm{x}_{\mathrm{i}}}\left(\Gamma_{\text {eff }} \frac{\partial \Phi}{\partial \mathrm{x}_{\mathrm{i}}}\right)+\mathrm{S}_{\Phi}
$$

And the overbar indicates the time-average values. In this way, it is not difficult to obtain the spatial distribution of the inclusion in the metallurgical reactor, but all the inclusions usually have the same size. Furthermore, the Eulerian approach has a fatal deficiency when it is applied to describe the inclusion's collision-aggregation. It ignored the fact that the inclusion's collision-growth in a non-uniform inclusion field is determined by the actual path, not the mean path the inclusion has passed.

For Lagrangian approach, ${ }^{2,3,7,8)}$ the governing equation for inclusion motion is based on Newton's second law, and usually is solved by Runge-Kutta-Gill method.

$$
\rho_{\mathrm{p}} \mathrm{V}_{\mathrm{p}} \frac{\mathrm{du}}{\mathrm{dt}}=\Sigma \overrightarrow{\mathrm{F}}
$$


In this way, it is easy to obtain the exact trajectory of single inclusion in the molten steel. But it is difficult to describe the collision-aggregation among inclusions and the spatial distribution of inclusions because there are too many inclusions to be traced in the current computational condition.

To overcome the deficiencies of the current Eulerian approach or Lagrangian approach, a Euler-Lagrange approach coupled with the Monte-Carlo method is developed to describe the inclusion's growth exactly. The related algorithm used in the present Fortran source code is shown in Fig. 1. The rest of paper will be organized as follows. Firstly, k- $\varepsilon$ turbulence model is applied to describe the three-dimensional flow field of molten steel. Secondly, the computation for the mass-population conservation model is performed to give the spatial distribution of inclusion cloud. Such an Eulerian approach is shown in section 2. Thirdly, the inclusion grows up on its travels in the inclusion cloud. For this propose, the continuous random walk model is introduced into the particle trajectory model to describe the effect of turbulent flow on the inclusion's motion, and the stochastic collision model and the adhension model are introduced into the traditional random rain model to study the effect of Stokes collision and turbulent collision on the inclusion's growth. Such a Lagrangian approach is shown in section 3. Section 4 will discuss the alumina particle behavior in a slab continuous casting mold. And the effect of turbulent flow on the inclusion's motion and collision-growth will be discussed in order to clarify the magnitude of the pressure gradient force, the visual mass force, the Basset history force, the Saffman

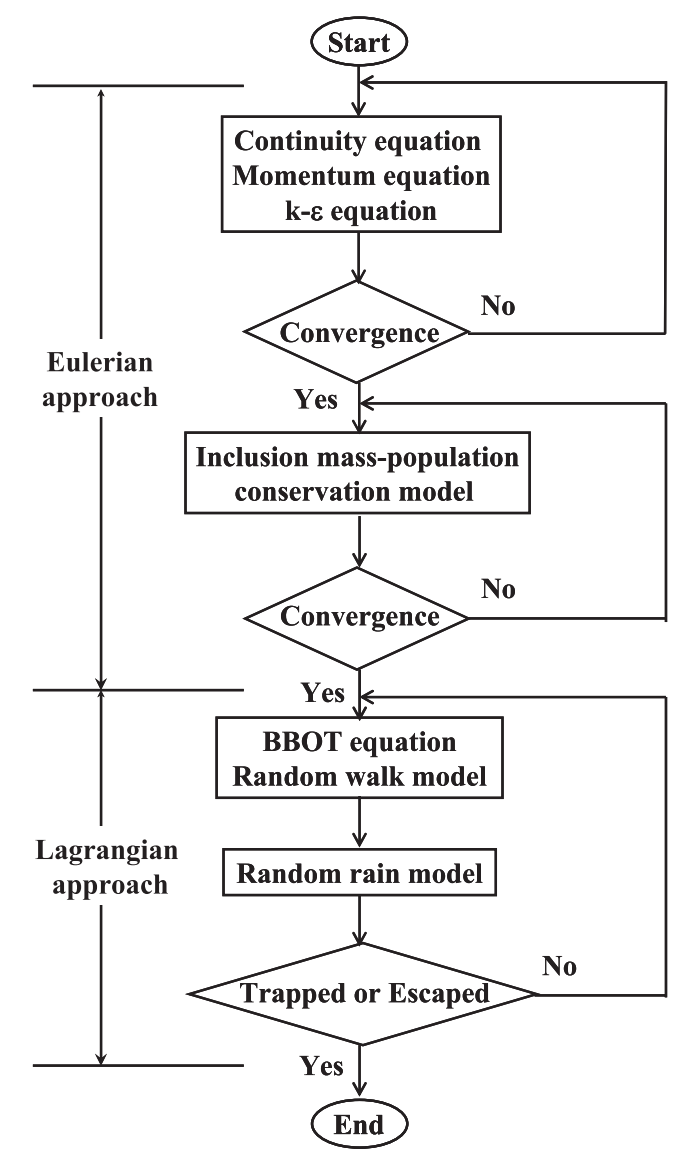

Fig. 1. Algorithm of Euler-Lagrange approach for transfer phenomena in the mold. lift force, the drag force, the buoyancy force and the gravitational force on inclusion's motion.

\section{Eulerian Method}

\subsection{Assumptions}

(1) The fluid flow in the mold is the steady and incompressible turbulent flow.

(2) The effect of the inclusion's movement on the fluid flow is negligible because their volume fraction is too low.

(3) The inclusion is a rigid spherical particles, and the entire cluster inclusion including the molten steel floats up at a uniform velocity.

(4) The inclusions will be removed from the molten steel if they reach the solidified shell.

(5) Some of the inclusions reaching the free surface are again entrained into the molten steel. And $80 \%$ of the inclusion flux which reaches the free surface is removed with the remaining $20 \%$ again entrained into the molten steel. ${ }^{10)}$

(6) The turbulent Schmidt number, $\mathrm{Sc}_{t}$, is equal to unity.

\subsection{Turbulent Flow}

Over the last twenty years, there have been numerous studies about the turbulent flow in the continuous caster because it affects many important phenomena that have farreaching consequences on strand quality. Among the turbulence models used nowadays, the standard k- $\varepsilon$ model is most widely used because it is efficient and gives reasonable results. The behavior of molten steel in the continuous casting mold can be described by the time-averaged mass conservation equation, the time-averaged momentum conservation equations and the standard k- $\varepsilon$ model. All these differential equations can be represented as the general Eq. (1).

The dimensions and operating parameters are listed in Table 1. Because of the symmetry, only half of the mold was considered in the present computation. The computational domain was analyzed by using a nonuniform grid of 50 (longitudinal) $\times 95$ (vertical) $\times 30$ (transverse) elements with a densely packed grid near the mold wall. Six types of boundaries enclosed the domain: the inlet, the outlet, the symmetry plane, the nozzle wall, the side wall and the free surface. And the detailed information can be found in the references. $^{2,12)}$ But it should be noted that the side wall is assumed to be the moving solidified shell with the casting

Table 1. Calculational and operating parameters for the slab caster.

\begin{tabular}{ll}
\hline \multicolumn{1}{c}{ Parameter/property } & \multicolumn{1}{c}{ Value } \\
\hline Nozzle entry & $85 \mathrm{~mm} \times 65 \mathrm{~mm}$, downward $15^{\circ}$ \\
Nozzle immersion depth & $300 \mathrm{~mm}$ \\
Slab cross-section & $1700 \mathrm{~mm} \times 220 \mathrm{~mm}$ \\
Calculation height & $3500 \mathrm{~mm}$ \\
Casting speed & $1.6 \mathrm{~m} / \mathrm{min}$ \\
$\mu$ & $0.0061 \mathrm{~Pa} \cdot \mathrm{s}$ \\
$\rho_{\mathrm{f}}$ & $7100 \mathrm{~kg} / \mathrm{m}^{3}$ \\
$\rho_{\mathrm{p}}$ & $3960 \mathrm{~kg} / \mathrm{m}^{3}$ \\
$\mathrm{C}^{*}$ & $120 \mathrm{ppm}$ \\
$\mathrm{r}^{*}$ & $5.8 \mathrm{ppm}$ \\
\hline
\end{tabular}


speed in the current model. ${ }^{13)}$ The governing equations associated with the boundary conditions are solved numerically by the finite-volume method. The convergence criterion are that the ratio of the difference between the input and output fluxes to input flux is less than $0.1 \%$ and the residual error of the mass source term is less than $10^{-7}$.

\subsection{Inclusion Cloud}

The non-uniform spatial distribution of inclusion is called as inclusion cloud. The inclusion has different size and different volumetric concentration at different position of the mold, so the inclusion size, the volumetric concentration and the number density are the key parameters to investigate the inclusion cloud in the mold.

\subsubsection{Governing Equations}

Industrial experiments show that the fractional inclusion number density decreases exponentially as the inclusion radius increases. ${ }^{10)}$

$$
\mathrm{f}(\mathrm{r})=\mathrm{Ae}^{-\mathrm{Br}}
$$

where A and B only depend on the coordinates. In this way, the inclusion number density and the inclusion volumetric concentration can be formulated as

$$
\begin{array}{r}
\mathrm{N}=\int_{0}^{\infty} \mathrm{f}(\mathrm{r}) \mathrm{dr}=\frac{\mathrm{A}}{\mathrm{B}} \\
\mathrm{C}=\int_{0}^{\infty} \frac{4}{3} \pi \mathrm{r}^{3} \mathrm{f}(\mathrm{r}) \mathrm{dr}=8 \pi \frac{\mathrm{A}}{\mathrm{B}^{4}}
\end{array}
$$

and the inclusion characteristic radius $\mathrm{r}^{*}$ can be formulated by

$$
r^{*}=\sqrt[3]{3 C /(4 \pi N)}=\frac{\sqrt[3]{6}}{B}
$$

Usually, the inclusion volume fraction is in the order of $0.01 \%$, so the inclusions' movement does not affect the macroscopic flow pattern of molten steel. In other words, the coupled calculation between the fluid flow and the inclusions' movement is one way. Consequently, on the base of the known flow field, the inclusion mass-population conservation model ${ }^{10)}$ was applied to predict the inclusion cloud with the assumption that the inclusion phase could be assumed as a continuum.

$$
\begin{aligned}
& \nabla \cdot\left(\rho_{\mathrm{f}} \overrightarrow{\mathrm{u}}_{\mathrm{C}^{*}} \mathrm{C}\right)=\nabla \cdot\left(\rho_{\mathrm{f}} \mathrm{D}_{\text {eff }} \nabla \mathrm{C}\right)+\mathrm{S}_{\mathrm{C}} \\
& \nabla \cdot\left(\rho_{\mathrm{f}} \overrightarrow{\mathrm{u}}_{\mathrm{N}} \mathrm{N}\right)=\nabla \cdot\left(\rho_{\mathrm{f}} \mathrm{D}_{\mathrm{eff}} \nabla \mathrm{N}\right)+\mathrm{S}_{\mathrm{N}}
\end{aligned}
$$

where the effective diffusion coefficient $\mathrm{D}_{\text {eff }}$ is expressed as

$$
\mathrm{D}_{\text {eff }}=\mathrm{D}_{0}+\frac{\mu_{\mathrm{t}}}{\rho \mathrm{Sc}_{\mathrm{t}}}
$$

The mass-population conservation model contains convective term, diffusion term and source term. The source term $\mathrm{S}_{\mathrm{C}}$ at the right hand of Eq. (8) depends on chemical reactions, and the source term $\mathrm{S}_{\mathrm{N}}$ at the right hand of Eq. (9) depends on chemical reactions and the inclusion's aggregation (or breakage). If there is no chemical reaction related to the inclusion in the metallurgic reactor, $\mathrm{S}_{\mathrm{C}}=0$, and $\mathrm{S}_{\mathrm{N}}$ will increases due to the inclusion's breakage or decreases due to the aggregation among the inclusions.
After a successful collision, two smaller inclusions disappear and a larger inclusion appears. The collision mechanisms for inclusions in molten steel include the turbulent collision, Stokes collision and Brownian collision. But the inclusion greater than $10 \mu \mathrm{m}$ is harmful to the product quality and Brownian collision is important only for the inclusion whose size less than $1 \mu \mathrm{m},{ }^{14)}$ thus only the turbulent collision and Stokes collision are considered in the present model.

$$
\begin{aligned}
& \beta_{\mathrm{ij}}^{\mathrm{St}}=\frac{2 \mathrm{~g} \pi}{9 \mu}\left(\rho_{\mathrm{f}}-\rho_{\mathrm{p}}\right)\left|\mathrm{r}_{\mathrm{i}}^{2}-\mathrm{r}_{\mathrm{j}}^{2}\right|\left(\mathrm{r}_{\mathrm{i}}+\mathrm{r}_{\mathrm{j}}\right)^{2} \\
& \beta_{\mathrm{ij}}^{\mathrm{t}}=1.3 \sqrt{\frac{\rho_{\mathrm{f}} \varepsilon}{\mu}}\left(\mathrm{r}_{\mathrm{i}}+\mathrm{r}_{\mathrm{j}}\right)^{3}
\end{aligned}
$$

In this way, the source term $\mathrm{S}_{\mathrm{N}^{*}}$ can be formulated by.

$$
\begin{aligned}
\mathrm{S}_{\mathrm{N}} & =\frac{1}{2} \int_{0}^{\infty} \int_{0}^{\infty}\left(\mathrm{P}_{\mathrm{t}} \beta_{\mathrm{ij}}^{\mathrm{t}}+\mathrm{P}_{\mathrm{St}} \beta_{\mathrm{ij}}^{\mathrm{St}}\right) \mathrm{N}_{\mathrm{i}} \mathrm{N}_{\mathrm{j}} \mathrm{dr}_{\mathrm{i}} \mathrm{dr}_{\mathrm{j}} \\
& =2.6 \mathrm{P}_{\mathrm{t}} \sqrt{\frac{\varepsilon}{v}} \mathrm{~N}^{2} \mathrm{r}^{* 3}+\frac{10}{9 \sqrt[3]{6}} \mathrm{P}_{\mathrm{St}} \frac{\pi \mathrm{g} \Delta \rho}{\mu} \mathrm{N}^{2} \mathrm{r}^{4}
\end{aligned}
$$

where $P_{t}$ is the adhension probability for turbulent collision, and $\mathrm{P}_{\mathrm{St}}$ is the adhension probability for Stokes collision.

The inclusion characteristic volumetric concentration velocity $\overline{\mathrm{u}}_{\mathrm{C}, \mathrm{i}}$ is the sum of the velocity of the fluid phase $\overline{\mathrm{u}}_{\mathrm{f}, \mathrm{i}}$ and the inclusion characteristic Stokes floatation velocity $\mathrm{u}_{\mathrm{C}, \mathrm{i}}^{\mathrm{St}}$ for the volumetric concentration equation. ${ }^{10)}$

$$
\overline{\mathrm{u}}_{\mathrm{C}, \mathrm{i}}=\overline{\mathrm{u}}_{\mathrm{f}, \mathrm{i}}+\mathrm{u}_{\mathrm{C}, \mathrm{i}}^{\mathrm{St}}
$$

Similarly, the inclusion characteristic number-density velocity $\overline{\mathrm{u}}_{\mathrm{N}, \mathrm{i}}$ is the sum of the velocity of the fluid phase $\overline{\mathrm{u}}_{\mathrm{f}, \mathrm{i}}$ and the inclusion characteristic Stokes floatation velocity $u_{N, i}^{S t}$ for the number density equation. ${ }^{10}$

$$
\overline{\mathrm{u}}_{\mathrm{N}, \mathrm{i}}=\overline{\mathrm{u}}_{\mathrm{f}, \mathrm{i}}+\mathrm{u}_{\mathrm{N}, \mathrm{i}}^{\mathrm{St}}
$$

The inclusion is modeled as a solid sphere, but there are many alumina clusters in the liquid steel in fact. Thus, the density and radius of a cluster inclusion should be the function of the volume fraction $\varphi$ of $\mathrm{Al}_{2} \mathrm{O}_{3}$ in the cluster. ${ }^{4)}$

$$
\begin{gathered}
\rho_{\mathrm{p}}=(1-\varphi) \rho_{\mathrm{f}}+\varphi \rho_{\mathrm{Al}_{2} \mathrm{O}_{3}} \\
\varphi \frac{4}{3} \pi \mathrm{r}_{\mathrm{p}}^{3}=\frac{4}{3} \pi \mathrm{r}_{\mathrm{Al}_{2} \mathrm{O}_{3}}^{3} .
\end{gathered}
$$

And the volume fraction of $\mathrm{Al}_{2} \mathrm{O}_{3}$ in the cluster is about $0.03{ }^{15)}$

\subsubsection{Boundary Conditions and Numerical Method}

The grid system for the inclusion transfer is the same as that for the fluid flow. The boundary condition can be found in the reference. ${ }^{10,11)}$ The partial differential equations for the inclusion's variables $\mathrm{C}$ and $\mathrm{N}$ were discretized by the finite-volume method, and the convergence criterion was $\max \left|\frac{\mathrm{C}^{(\mathrm{K}+1)}-\mathrm{C}^{(\mathrm{K})}}{\mathrm{C}^{(\mathrm{K}+1)}}\right|<10^{-8}$, where $\mathrm{K}$ is the iteration number.

\section{Lagrangian Approach}

\subsection{Assumption}

(1) The inclusion does not rotate during its motion. 
(2) Every inclusion moves independently until two inclusions collide.

(3) The inclusions are released at the outlet of the submerged nozzle randomly. The initial locations are uniformly distributed within the exit of the submerged entry nozzle, and their initial velocities are equal to the local fluid velocity.

(4) The calculation will not stop until the inclusion is trapped by the solidifying-shell (or the slag), or is escaped from the outlet.

(5) The removed probability is only $80 \%$ if the inclusion reaches the free surface, and it is $100 \%$ if the inclusion is trapped by the solidifying-shell or the slag. ${ }^{10)}$

(6) The turbulence eddy has the sphere shape.

\subsection{Particle Trajectory Model}

All the time-average data of flow field, including fluid velocity, turbulent kinetic energy, dissipation rate of turbulent kinetic energy, inclusion number density and inclusion volumetric concentration can be known a priori. The effect of the turbulence on the inclusion movement was considered, but the inclusion concentration is so low that the effect of particle movement on the fluid turbulence can be ignored.

The motion of inclusions in the molten steel can be described in a Lagrangian way by solving a set of ordinary differential equations along the trajectory in order to calculate the change of inclusion location. This model needs to consider all the relevant forces acting on the inclusion, and the equation of particle motion is mostly referred to as BassetBoussinesq-Oseen-Tchen(BBOT) equations.

$$
\rho_{\mathrm{p}} \mathrm{V}_{\mathrm{p}} \frac{\mathrm{d} \overrightarrow{\mathrm{u}}_{\mathrm{p}}}{\mathrm{dt}}=\overrightarrow{\mathrm{F}}^{\mathrm{d}}+\overrightarrow{\mathrm{F}}^{\mathrm{g}}+\overrightarrow{\mathrm{F}}^{\mathrm{b}}+\overrightarrow{\mathrm{F}}^{\mathrm{v}}+\overrightarrow{\mathrm{F}}^{\mathrm{B}}+\overrightarrow{\mathrm{F}}^{\mathrm{P}}+\overrightarrow{\mathrm{F}}^{\mathrm{S}}
$$

where $V_{p}$ is the volume of the spherical inclusion and $\rho_{\mathrm{p}}$ is the particle density.

Here, the drag force comes from the relative velocity of the inclusion and the molten steel.

$$
\overrightarrow{\mathrm{F}}^{\mathrm{d}}=\frac{1}{2} \cdot \frac{1}{4} \pi \mathrm{d}_{\mathrm{p}}^{2} \rho_{\mathrm{f}} \mathrm{C}_{\mathrm{D}}\left|\overrightarrow{\mathrm{u}}_{\mathrm{f}}-\overrightarrow{\mathrm{u}}_{\mathrm{p}}\right|\left(\overrightarrow{\mathrm{u}}_{\mathrm{f}}-\overrightarrow{\mathrm{u}}_{\mathrm{p}}\right) \ldots \ldots
$$

with

$$
C_{D}=\frac{24}{\operatorname{Re}_{p}}
$$

and

$$
\operatorname{Re}_{\mathrm{p}}=\frac{\rho_{\mathrm{f}} \mathrm{d}_{\mathrm{p}}\left|\overrightarrow{\mathrm{u}}_{\mathrm{p}}-\overrightarrow{\mathrm{u}}_{\mathrm{f}}\right|}{\mu_{\mathrm{f}}}
$$

The gravitational force causes the inclusion to descend in the molten steel.

$$
\overrightarrow{\mathrm{F}}^{\mathrm{g}}=\frac{1}{6} \pi \mathrm{d}_{\mathrm{p}}^{3} \rho_{\mathrm{p}} \overrightarrow{\mathrm{g}}
$$

Similarly, the buoyancy force causes the inclusion to rise in the molten steel.

$$
\overrightarrow{\mathrm{F}}^{\mathrm{b}}=\frac{1}{6} \pi \mathrm{d}_{\mathrm{p}}^{3} \rho_{\mathrm{f}} \overrightarrow{\mathrm{g}}
$$

The visual mass force (or the added force) is a result of the molten steel surrounding the inclusion being accelerated. It has a tendency to keep the inclusion from being accelerated in any direction.

$$
\overrightarrow{\mathrm{F}}^{\mathrm{v}}=\frac{1}{2} \rho_{\mathrm{f}} \mathrm{V}_{\mathrm{p}}\left(\frac{\mathrm{d} \overrightarrow{\mathrm{u}}_{\mathrm{f}}}{\mathrm{dt}}-\frac{\mathrm{d} \overrightarrow{\mathrm{u}}_{\mathrm{p}}}{\mathrm{dt}}\right)
$$

The Basset history force is the force associated with past movements of the particle.

$$
\overrightarrow{\mathrm{F}}^{\mathrm{B}}=\frac{3}{2} \mathrm{~d}_{\mathrm{p}}^{2} \sqrt{\pi \rho_{\mathrm{f}} \mu_{\mathrm{f}}} \int_{0}^{\mathrm{t}} \frac{\frac{\mathrm{d} \overrightarrow{\mathrm{u}}_{\mathrm{f}}}{\mathrm{d} \tau}-\frac{\mathrm{d} \overrightarrow{\mathrm{u}}_{\mathrm{p}}}{\mathrm{d} \tau}}{\sqrt{\mathrm{t}-\tau}} \mathrm{d} \tau
$$

The pressure gradient force comes from the acceleration of the molten steel.

$$
\overrightarrow{\mathrm{F}}^{\mathrm{P}}=\rho_{\mathrm{f}} \mathrm{V}_{\mathrm{d}} \frac{\mathrm{d} \overrightarrow{\mathrm{u}}_{\mathrm{f}}}{\mathrm{dt}}
$$

The Saffman's lift force becomes large where the velocity gradient of molten steel is large.

$$
\overrightarrow{\mathrm{F}}^{\mathrm{S}}=1.61 \mathrm{~d}_{\mathrm{p}}^{2}\left(\mu_{\mathrm{f}} \rho_{\mathrm{f}}\right)^{1 / 2}\left|\nabla \times \overrightarrow{\mathrm{u}}_{\mathrm{f}}\right|^{-1 / 2}\left[\left(\overrightarrow{\mathrm{u}}_{\mathrm{f}}-\overrightarrow{\mathrm{u}}_{\mathrm{p}}\right) \times\left(\nabla \times \overrightarrow{\mathrm{u}}_{\mathrm{f}}\right)\right]
$$

The inclusion trajectory Eq. (16), which was solved by stepwise integration over discrete time steps, gives the velocity of the inclusion at each point along with its trajectory. In this way, the inclusion trajectories can be computed by

$$
\frac{\mathrm{d} \overrightarrow{\mathrm{x}}_{\mathrm{p}}}{\mathrm{dt}}=\overrightarrow{\mathrm{u}}_{\mathrm{p}}
$$

where $\vec{x}_{p}$ is the location of the inclusion particle.

\subsection{Particle Random Walk Model}

For Lagrangian method, how to specify the ever-changing flow field $u_{\mathrm{f}}$ is one of the important problems related to inclusion movement in turbulent flow. The simplest way is the introduction of the eddy life-time model, ${ }^{7,8)}$ which use a Gaussian probability distribution for fluctuating velocity $\mathrm{u}_{\mathrm{f}, \mathrm{i}}^{\prime}$ with a mean value of zero and the root mean square fluctuating velocity $\overline{\mathrm{u}_{\mathrm{f}, \mathrm{i}}^{\prime 2}}$ as standard deviation. In other words, the fluctuating velocity components are computed stochastically by a Monte-Carlo method.

$$
\mathrm{u}_{\mathrm{f}, \mathrm{i}}=\overline{\mathrm{u}}_{\mathrm{f}, \mathrm{i}}+\mathrm{u}_{\mathrm{f}, \mathrm{i}}^{\prime}=\overline{\mathrm{u}}_{\mathrm{f}, \mathrm{i}}+\xi \sqrt{2 \mathrm{k} / 3}
$$

In this model, the inclusion interacts with the molten steel in the turbulent eddies over the eddy life-time $\tau_{\mathrm{f}}$. When the eddy life-time is reached, a new value of the fluid instantaneous velocity is obtained by applying a new value of random number $\xi$, whose value is uniformly distributed between -1 and 1 . Each eddy is characterized by a Gaussian distributed random velocity fluctuation $\mathrm{u}_{\mathrm{f}, \mathrm{i}}^{\prime}$, which keeps the constant over the characteristic life-time of the eddies.

In the eddy life-time model, the flow field is assumed to be frozen during each time interval. But in fact, the velocities at adjacent points or the velocities at a point at subsequent instants are not perfectly random but are inter-related, so the assumption of frozen flow field may lead to some errors. However, these shortcomings will be improved if these correlation functions between adjacent points or between subsequent intervals can be taken into account in the mathematical model.

The continuous random walk model proposed by $\mathrm{Lu}^{16,17)}$ can avoids the above shortcomings effectively, because the crossing trajectory and continuity effects are clearly 


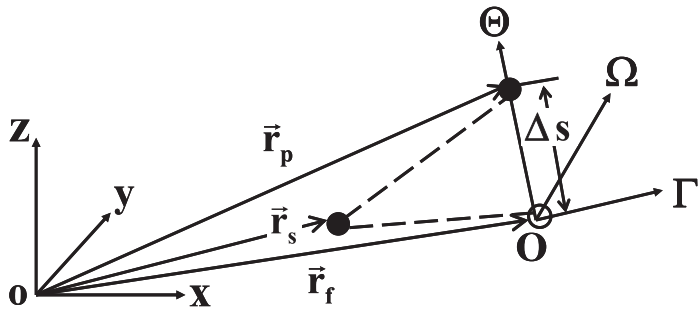

Fig. 2. Sketch of the velocity correlations simulation.

observed and the particle diffusion coefficients are close to the theoretical values.

In order to determine the correlation between the new fluctuating velocity and the fluctuating velocity at the previous step, the movement of a fluid particle and the inclusion particle during one time step is shown in Fig. 2, At the instant $t$, an inclusion particle and a fluid particle start out from the same position $\vec{r}_{\mathrm{s}}$. After one time step, $\Delta \mathrm{t}$, the inclusion moves to a news location $\vec{r}_{p}$, which is different from the new position of fluid particle $\vec{r}_{\mathrm{f}}$. And the distance between the new positions is expressed as $\Delta \mathrm{s}$.

Here, o-xyz system is the absolute coordinate system, and $\mathrm{O}-\Gamma \Omega \Theta$ system is the relative coordinate system based on $\Delta$ s. The new fluctuating velocity of the molten steel at the position $\vec{r}_{\mathrm{p}}$ has a linear relationship with the preceding fluctuation velocity at the position $\vec{r}_{\mathrm{s}}$. This is called a Markov chain.

$$
\mathrm{W}_{\mathrm{i}}\left(\overrightarrow{\mathrm{r}}_{\mathrm{p}}\right)=\mathrm{f}(\Delta \mathrm{t}) \mathrm{g}_{\mathrm{i}}(\Delta \mathrm{s}) \mathrm{W}_{\mathrm{i}}\left(\overrightarrow{\mathrm{r}}_{\mathrm{s}}\right)+\psi_{\mathrm{i}} \quad(\mathrm{i}=1,2,3) \ldots .
$$

where the normalized fluctuating component is defined on the $\mathrm{O}-\Gamma \Omega \Theta$ coordinate system.

$$
\mathrm{W}_{\mathrm{i}}=\frac{\mathrm{u}_{\mathrm{i}}^{\prime \mathrm{f}}}{\sqrt{\overline{\mathrm{u}_{\mathrm{i}}^{\prime \mathrm{f}} \mathrm{u}_{\mathrm{i}}^{\prime \mathrm{f}}}}} \quad(\mathrm{i}=1,2,3)
$$

Here, the subscripts 1,2 and 3 denote the directions of $\Gamma, \Omega$ and $\Theta$ axis in Fig. 2, and the overbars indicate the ensemble average values. The random variables $\psi_{\mathrm{i}}$ is assumed to be a Gaussian probability distribution with a mean value of zero and a standard variance $\left(\sigma_{\psi}\right)_{\mathrm{i}}$ which are given by

$$
\left(\sigma_{\psi}\right)_{\mathrm{i}}=\sqrt{1-\mathrm{f}^{2}(\Delta \mathrm{t}) \mathrm{g}_{\mathrm{i}}^{2}(\Delta \mathrm{s})} \quad(\mathrm{i}=1,2,3)
$$

In this way, the velocity fluctuations of molten steel along with the inclusion's trajectory can be reduced from the correlation functions of the molten steel. Consequently, the inhomogeneity of turbulence and the inclusion drift can be described without further correction.

For the implementation of this model, it is necessary to give an appropriate expression for the correlation functions $\mathrm{f}(\Delta \mathrm{t})$ and $\mathrm{g}_{\mathrm{i}}(\Delta \mathrm{s})$.

$$
\begin{array}{r}
\mathrm{f}(\Delta \mathrm{t})=\exp \left(\frac{-\Delta \mathrm{t}}{\tau_{\mathrm{f}}}\right) \ldots \ldots \ldots \ldots \ldots \ldots \ldots \ldots \ldots \ldots \ldots \ldots . . . \\
\mathrm{g}_{\mathrm{i}}(\Delta \mathrm{s})=\exp \left(\frac{-\Delta \mathrm{s}}{2 \Lambda_{\mathrm{i}}}\right) \cos \left(\frac{\Delta \mathrm{s}}{2 \Lambda_{\mathrm{i}}}\right) \quad(\mathrm{i}=1,2,3) \ldots
\end{array}
$$

The Lagrangian integral timescales $\tau_{\mathrm{f}}$ and the Eulerian length-scale $\Lambda_{\mathrm{i}}$ can be deduced from the statistical turbulence quantities in the standard k- $\varepsilon$ model.

$$
\tau_{\mathrm{f}}=0.157 \frac{\mathrm{k}}{\varepsilon}
$$

$$
\begin{aligned}
& \Lambda_{1}=2.04 \tau_{\mathrm{f}} \sqrt{\mathrm{k}} \\
& \Lambda_{2}=\Lambda_{3}=0.5 \Lambda_{1}
\end{aligned}
$$

Here, $\Lambda_{1}$ is the longitudinal length-scale, and $\Lambda_{2}$ and $\Lambda_{3}$ are transverse length-scales.

\subsection{Random Rain Model in the Turbulent Flow}

In the continuous casting mold, the turbulent flow is the key factor to affect the inclusion's movement and the inclusion's collision-growth. Section 3.3 gives one of the good solutions for the effect of the turbulence on the particle's trajectory. The remained problem can be solved by seeking help from the fractal geometry theory, a powerful tool to describe the non-equilibrium growth process. The traditional random rain model ${ }^{18)}$ can simulate the cluster's growth process, but the parameters(the length and the time) is only the mathematical variables and have nothing to do with the physical background. Thus, the stochastic collision model by O'Rourke ${ }^{19)}$ and the adhension model in chemical engineering ${ }^{20,21)}$ are introduced to be a bridge from the random rain model to the cluster inclusion's collision-aggregation model. In this way, the modified random rain model has to solve the following three problems: the collision between two inclusions, the adhension between two inclusions, and the adhension point between two inclusions.

The collision-aggregation is a stochastic process. And the overall collision-aggregation probability is the product of collision probability $\mathrm{P}_{c o l}$ and the adhension probability $\mathrm{P}_{\mathrm{adh}}$.

$$
\mathrm{P}_{\text {oca }}=\mathrm{P}_{\text {col }} \cdot \mathrm{P}_{\text {adh }}
$$

\subsubsection{Collision Probability}

The first issue arising is how to determine the occurrence of a collision between two inclusions. The tracked inclusion with radius $r_{j}$ is called as the seed inclusion, another shot inclusion should be chosen at random. Here, the appearance probability for the shot inclusion with radius $r_{i}$ to collide with the seed inclusion with radius $r_{j}$ can be formulated by

$$
P\left(r_{i}\right)=\frac{\int_{r_{i}}^{r_{i+1}} f(r) d r}{\int_{0}^{\infty} f(r) d r}
$$

According to the geometry, the collision occurs if the distance between two inclusions is less than $r_{i}+r_{j}$. In this way, the expected collision number between the seed inclusion and the shot inclusion ${ }^{19)}$ can be expressed as

$$
\overline{\mathrm{m}}=\frac{\mathrm{N}_{\mathrm{j}} \pi\left(\mathrm{r}_{\mathrm{i}}+\mathrm{r}_{\mathrm{j}}\right)^{2} \mathrm{v} \Delta \mathrm{t}}{\mathrm{V}}
$$

where $\mathrm{v} \Delta \mathrm{t}$ is the distance traveled by the seed particle, $\mathrm{V}$ is the volume of the mesh cell.

And the probability distribution of the collision number among inclusions follows the Poisson distribution. ${ }^{19)}$

$$
\mathrm{P}_{\mathrm{col}}(\mathrm{m})=\frac{\overline{\mathrm{m}}^{\mathrm{m}}}{\mathrm{m} !} \exp (-\overline{\mathrm{m}})
$$

\subsubsection{Adhension Probability}

The adhension between two inclusions is the second step for two inclusions to form a bigger inclusion. Such a phe- 
nomenon depends on the adhensive force and the hydrodynamic interactions.

Turbulent collision and Stokes collision among inclusions are the main collision mechanisms for inclusions to grow up, and different collisions have different adhension probability. Thus, it is necessary to determine the collision mechanism between two inclusions at first.

$$
\mathrm{P}_{\mathrm{adh}}= \begin{cases}\mathrm{P}_{\mathrm{St}} & 0 \leq \zeta \leq \frac{\beta_{\mathrm{St}}}{\beta_{\mathrm{St}}+\beta_{\mathrm{t}}} \\ \mathrm{P}_{\mathrm{t}} & \frac{\beta_{\mathrm{St}}}{\beta_{\mathrm{St}}+\beta_{\mathrm{t}}}<\zeta \leq 1\end{cases}
$$

where the random variable $\zeta$ is assumed to follow the uniform distribution with mean value 0.5 .

If the collision between two inclusions is turbulent collision, the related adhension probability ${ }^{20,21)}$ can be formulated by

$$
\mathrm{P}_{\mathrm{t}}=0.732\left(\frac{5}{\mathrm{~N}_{\mathrm{T}}}\right)^{0.242}
$$

where $\mathrm{N}_{\mathrm{T}}$ is the ratio of the viscous force and the Van der Walls force.

And the adhension probability due to Stokes is unity in the existed references. ${ }^{10,14)}$

$$
\mathrm{P}_{\mathrm{S}}=1
$$

\subsubsection{Adhension Position}

The adhension position can be determined on the base of the random rain model. The detailed information is described as follows:

(1) The seed inclusion with radius $r_{j}$ is place at the center of turbulent eddy.

(2) The radius $r_{j}$ of the shot inclusion is determined by Eq. (36).

(3) The shot inclusion starts inward from the surface of the sphere.

(4) If the collision between two inclusions occurs, the collision position is accepted as the adhension point. If not, return to step (2), and repeat the whole procedure until the collision occurs.

\subsection{Numerical Method}

A fourth-order Runge-Kutta-Gill method was adopted to solve these equations simultaneously. The time step $\Delta \mathrm{t}$, which is taken to calculate both the inclusion trajectory equation and the modified random rain model, is related to the grid system and the characteristics of turbulent flow.

$$
\Delta \mathrm{t}=\frac{\min \left(\tau_{\text {grid }}, \tau_{\mathrm{f}}\right)}{\mathrm{c}_{\mathrm{t}}}
$$

with $\tau_{\text {grid }}=\min \left(\Delta \mathrm{x}_{\mathrm{i}} / \mathrm{u}_{\mathrm{f}, \mathrm{i}}\right)(\mathrm{i}=1,2,3)$ and where $\mathrm{c}_{\mathrm{t}}>10$ is the time-step length factor.

\section{Results and Discussion}

Figure 3 presents the predicted fluid flow in the continuous casting mold. The stream from the nozzle spread out until it impinges against the narrow wall, and then forms the upper recirculation zone caused by the upward stream and the lower recirculation zone caused by the downward stream.

Figure 4 shows the inclusion cloud covers all the places in the mold due to the turbulent flow, and the spatial distribution of the inclusion volumetric concentration, the inclusion number density and the inclusion characteristic radius

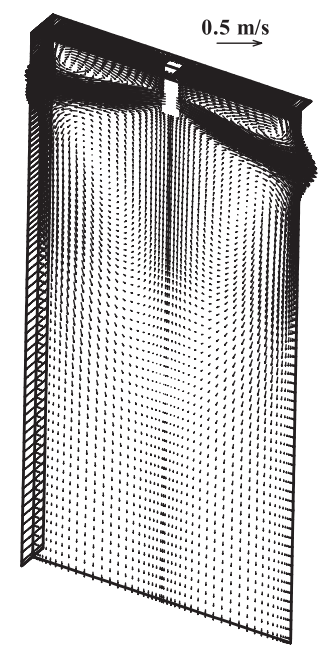

Fig. 3. Fluid flow in the mold.

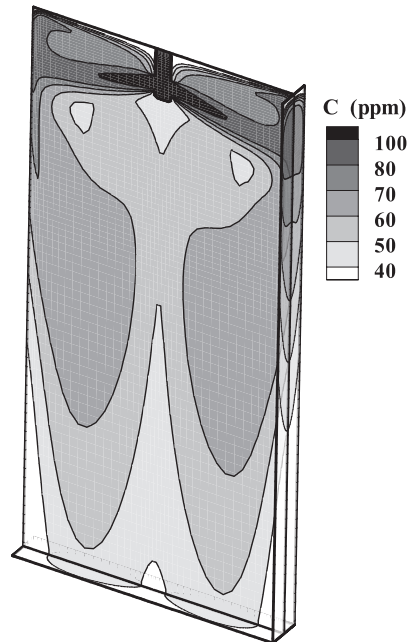

(a) $\mathrm{C}$

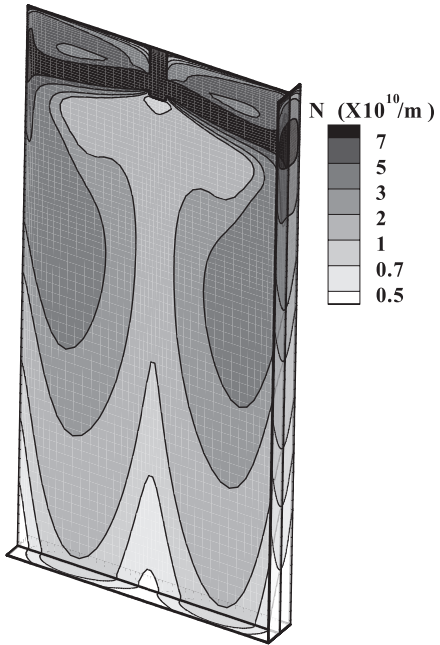

(b) $\mathrm{N}$

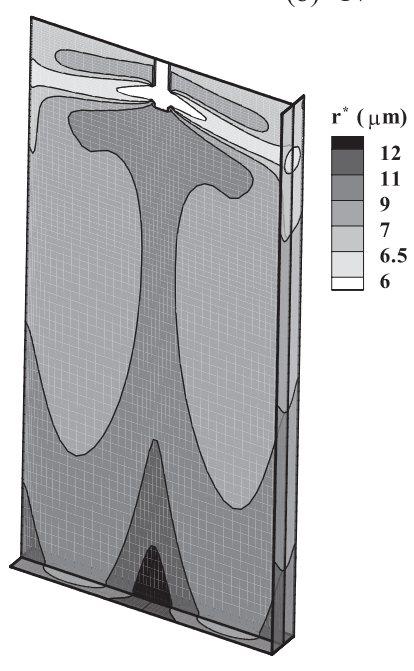

(c) $r^{*}$

Fig. 4. Inclusion cloud in the mold. 
has the 'W' shape near the exit. Such an interesting distribution has been observed in Javureks's experiment. ${ }^{9)}$ The darker part in the Figs. 4(a) and 4(b) indicates that there are the greater inclusion volumetric concentration and the greater inclusion number density. Thus, the concept of inclusion cloud is introduced to describe the uneven inclusion spatial distribution. Such an uneven inclusion cloud distribution comes from the follows reasons: the inclusion removal by the top slag, the inclusion capture by the solidified shell, the inclusion collision-growth and the inclusion transfer by the fluid flow.

The fluid flow is the key factor to affect the inclusion spatial distribution. Similar to the fluid flow, the inclusion volumetric concentration, the inclusion number density and the inclusion characteristic radius also have some features of the upper recirculation zone and the lower recirculation zone, as shown in Figs. 3 and 4. And at the center of the recirculation zone, the inclusion volumetric concentration and the inclusion number density are smaller, and the inclusion characteristic radius is greater. Several reasons lead to this interesting phenomenon. (1) The velocity of the molten steel is very slow near the center of the recirculation zone, so the molten steel can not be refreshed in time. (2) For the inclusion in the fresh liquid steel, the inclusion volumetric concentration and the inclusion number density are greater, but the inclusion size is small. (3) The velocity of molten steel is far greater than the inclusion Stokes floatation velocity, so the inclusion transport is controlled by the fluid flow. (4) The inclusions grows up because of the collision-aggregation among the inclusions.

Another interesting phenomenon is that the inclusion's distribution has ' 8 ' shape structure at the exit. Especially for the inclusion characteristic radius, the larger inclusions are close to the slab surface, which was observed by Kumar, ${ }^{22)}$ and the biggest inclusions appear at the center of the liquid pool. These interesting phenomena depend on the following factors. (1) The velocity of molten steel have ' $\mathrm{W}$ ' shape distribution at the longitudinal direction and ' $\mathrm{V}$ ' shape distribution at the traverse direction due to the wall effect of the slab shell. (2) Near the shell, the molten steel can not be refreshed in time because the molten steel flow slowly, but the inclusions collide and aggregate continuously. (3) At the center of the liquid pool, the inclusions have more time to collide and aggregate because the molten steel flows slowly. (4) Some inclusions are trapped by the slab shell.

Due to the entrainment of the molten steel, the inclusion follows the movement of molten steel. Figure 5 shows that the upper and lower recirculation structure of flow field results in the inclusion's loop motion. After the loop motion, part of the inclusions float up and are removed by the slag, part of the inclusions are trapped by the solidified shell at the narrow face (or wide face) of the slab, and other inclusions enter the liquid pool. It should be noted that inclusion's trajectories are not the smooth curves but the zigzag curves. Such a phenomenon comes from the effect of the turbulent flow on the inclusion's movement. In other words, the fluctuation is the leading feature of the turbulence, so the particle changes its direction of motion frequently.

Figure 6 shows that the aggregated inclusion, which is called the cluster-shape inclusion, has one or several chains. Some similar inclusions were observed by Yin $^{23)}$ and

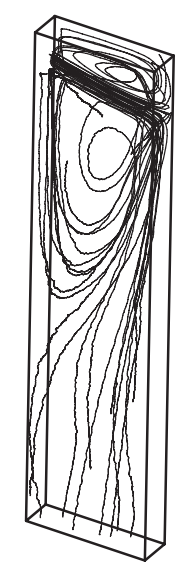

Fig. 5. Predicted inclusions' trajectories in the mold.

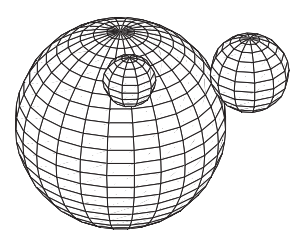

(a) $10 \mu \mathrm{m}$

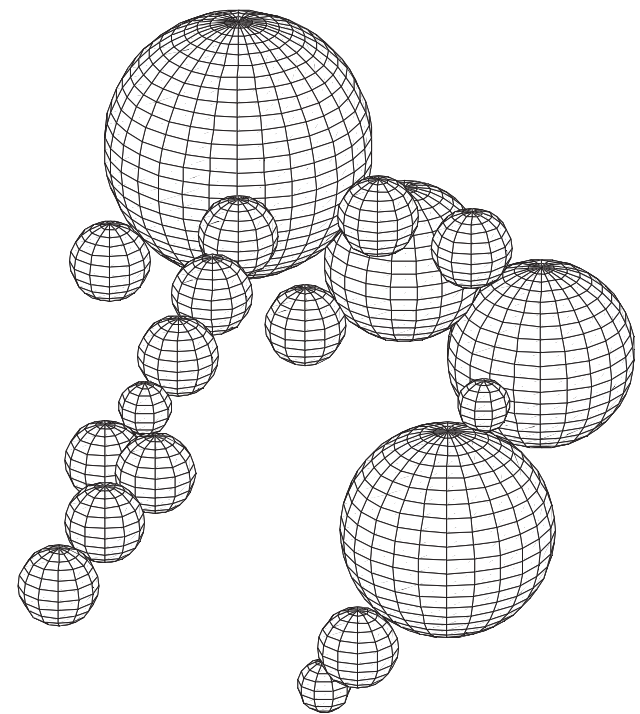

(b) $50 \mu \mathrm{m}$

Fig. 6. Cluster-like inclusions formed from different seed inclusions. 
Mizoguch. ${ }^{24)}$ And the bigger seed inclusion has the more complex cluster structure. Several reasons lead to this phenomenon. (1) During the seed inclusion's movement, it passes by different clouds. The darker cloud about inclusion number density means that there are more inclusions so the seed inclusion has more chances to collide with other inclusions. (2) The big inclusion, which has a big surface, has more chances to catch other inclusions and becomes a bigger inclusion. This is a vicious cycle. (3) The starting position, the inclusion's movement and the collision-aggregation among the inclusions are stochastic processes. (4) After the seed inclusion caught the new inclusion, a sharp tip appears in the seed inclusion's periphery. Consequently, it is easier for the seed inclusion to catch other inclusions because of the larger surface. Finally, these extended tips become the chains.

Figure 7 shows that the variation of different forces acting on the inclusion along with its own trajectory, and give the following rules. (1) The gravitational force is almost equal to the buoyancy force comes from such a fact that the density of cluster inclusion is very close to that of the molten steel because the volume fraction of alumina in the cluster is $0.03 .^{15)}(2)$ The pressure gradient force is the most important force during the inclusion's movement because it is about one order greater than the gravitational force. Such a phenomenon comes from the fact that the inclusion particle has to follow the fluid flow because the inclusion size is very small. And the fluctuation velocity is the characteristics of the turbulent flow. In this case, the pressure gradient force acts as the key drive force for the inclusion's movement. (3) The second important force is the Basset history force, which is about three times greater than the gravitational force. (4) The gravitational force, the buoyancy force, the visual mass force and the drag force are at the same order. (5) The Saffman lift force is about two orders less than the gravitational force because the velocity gradient of molten steel is very small in the mold. (5) The pressure gradient force, the Basset history force, the visual force and the drag force follow the same variation rule along with the inclusion's trajectory although they are not in the same order. Two reasons lead to this interesting phenomenon. Firstly, the acceleration of the fluid is the key factor to affect the pressure gradient force, the Basset history force and the visual

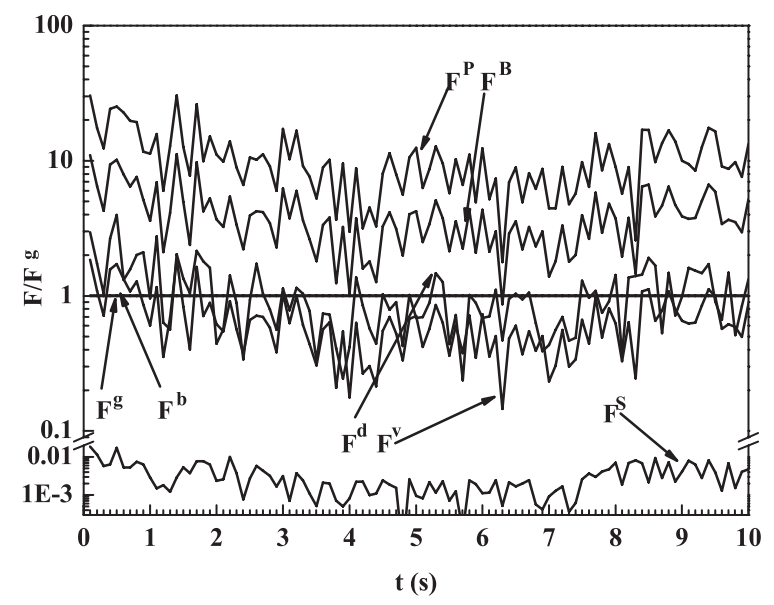

Fig. 7. Forces acting on $10 \mu \mathrm{m}$ inclusion along with its trajectory. force. Secondly, the driving force (the pressure gradient force) control the inclusion's movement and is one order higher than the passive force (the drag force), so the drag force have to follow the variation of the pressure gradient force.

\section{Conclusions}

In order to describe the inclusion collision-growth process in the continuous casting mold, the Euler-Lagrange approach was applied to describe the forming process of the cluster-structure inclusion by tracing an inclusion movement in the inclusion cloud. In this model, the turbulent collision and Stokes collision are the key collision mechanisms, and the modified random rain model in the fractal theory has the clear physical background. The related results can be summed up as follows:

(1) The fluid flow is the key factor to affect the inclusion spatial distribution in the mold.

(2) The inclusion's zigzag trajectory comes from the interaction between turbulent flow and the inclusion.

(3) The bigger inclusion has more chances to become a cluster inclusion.

(4) In order to describe the exact motion of an inclusion, the pressure gradient force, the Basset history force, the drag force, the visual mass force, the buoyancy force and the gravitational force should be considered, and the Saffman lift force can be ignored because it is two orders less than the gravitational force.

(5) The pressure gradient force, the Basset history force, the visual mass force follow the same variation rule along with the particle's trajectory.

\section{Acknowledgements}

This work was supported by the National Natural Science Foundation of China (51304038), Liaoning BaiQianWan Talents Program (2013921073), Open Funds of the State Key Laboratory of Advanced Metallurgy at the University of Science and Technology of Beijing (KF13-10), the China Postdoctoral Science Foundation (2013M530130) and the Postdoctoral Science Foundation of Northeastern University.

\section{Nomenclatures}

A parameter depending on the coordinate, $1 / \mathrm{m}^{4}$

B parameter depending on the coordinate, $1 / \mathrm{m}$

C inclusion volumetric concentration, (-)

$\mathrm{C}_{\mathrm{D}}$ drag coefficient, (-)

D diffusion coefficient, $\mathrm{m}^{2} / \mathrm{s}$

f fractional inclusion number density, $1 / \mathrm{m}^{4}$

$\mathrm{f}(\Delta \mathrm{t})$ time correlation function

$\mathrm{F}$ force, $\mathrm{N}$

g gravitational acceleration, $\mathrm{m} / \mathrm{s}^{2}$

$\mathrm{g}(\Delta \mathrm{s})$ length correlation function

$\mathrm{k}$ turbulent energy, $\mathrm{m}^{2} / \mathrm{s}^{2}$

$\mathrm{K}$ iteration time, $(-)$

$\mathrm{L}$ length, $\mathrm{m}$

$\mathrm{m}$ collision number

$\mathrm{N}$ inclusion number density, $1 / \mathrm{m}^{3}$

$\mathrm{N}_{\mathrm{T}}$ ratio of the viscous force and the Van der Walls force, $(-)$

P probability 
$\mathrm{r}$ radius, $\mathrm{m}$

$r^{*}$ inclusion characteristic radius, $m$

$\mathrm{Re}_{\mathrm{p}}$ particle Reynolds number, (-)

$\mathrm{S}_{\mathrm{C}}$ source term in the inclusion volumetric concentration equation, $\mathrm{kg} /\left(\mathrm{m}^{3} \cdot \mathrm{s}\right)$

$\mathrm{Sc}_{\mathrm{t}}$ Schmidt number

$\mathrm{S}_{\mathrm{N}}$ source term in the inclusion volumetric number density equation, $\mathrm{kg} /\left(\mathrm{m}^{6} \cdot \mathrm{s}\right)$

$\mathrm{t}$ time, $\mathrm{s}$

$\mathrm{u}$ velocity, $\mathrm{m} / \mathrm{s}$

$\mathrm{V}$ volume, $\mathrm{m}^{3}$

W normalized fluctuating velocity, (-)

$\mathrm{x}, \mathrm{y}, \mathrm{z}$ coordinate, $\mathrm{m}$

\section{Greek symbols}

$\beta$ collision rate, $\mathrm{m}^{3} / \mathrm{s}$

$\varepsilon$ turbulent energy dissipation rate, $\mathrm{m}^{2} / \mathrm{s}^{3}$

$\varphi$ volume fraction, (-)

$\Gamma$ coordinate, $\mathrm{m}$

$\mu$ dynamic viscosity, $\mathrm{Pa} \cdot \mathrm{s}$

$\rho$ density, $\mathrm{kg} / \mathrm{m}^{3}$

$\Omega$ coordinate, $\mathrm{m}$

$\sigma_{\psi} \quad$ standard variance

$\Lambda$ length-scale

$\tau$ eddy life time, $\mathrm{s}$

$\Theta$ coordinate, $m$

$\xi, \psi$ random number, (-)

\section{Subscripts}

$\begin{aligned} \text { adh } & \text { adhension } \\ \mathrm{b} & \text { buoyancy } \\ \mathrm{B} & \text { Basset } \\ \mathrm{Br} & \text { Brownian } \\ \mathrm{col} & \text { collision } \\ \mathrm{d} & \text { drag } \\ \text { eff } & \text { effective } \\ \mathrm{f} & \text { fluid } \\ \mathrm{g} & \text { gravity } \\ \mathrm{i}, \mathrm{j} & \text { index }\end{aligned}$

oca overall collision-aggregation

$\mathrm{p}$ inclusion particle

$\mathrm{P}$ pressure gradient

S Saffman lift

St Stokes

$\mathrm{t}$ turbulent

$\mathrm{v}$ visual mass

\section{REFERENCES}

1) S. Joo and I. L. Guthrie: Metall. Trans. B, 24 (1993), 755.

2) Y. H. Ho and W. S. Hwang: ISIJ Int., 43 (2003), 1715.

3) B. Li and F. Tsukihashi: ISIJ Int., 43 (2003), 923.

4) Y. Miki, B. G. Thomas, A. Denissov and Y. Shimada: Iron Steelmaker, 24 (1997), 31.

5) H. Lei and J. He: J. Mater. Sci. Technol., 28 (2012), 642.

6) H. Li, L. Ning, J. Wen, J. Zhang, Y. Sasaki and M. Hino: J. Univ. Sci. Technol. Bejing, 13 (2006), 117.

7) G. Solorio-Diaz, R. D. Morales, J. Palafox-Ramos and A. RamosBanderas: ISIJ Int., 45 (2005), 1129.

8) S. Lopez-Ramirez, J. J. Barreto, J. Palafox-Ramos, R. D. Morales and D. Zacharias: Metall. Mater. Trans. B, 32 (2001), 615.

9) M. Javurek, P. Gittler, R. Rossler, B. Kaufmann and H. Preblinger: Steel Res. Int., 76 (2005), 64.

10) H. Lei, L. Wang, Z. Wu and J. Fan: ISIJ Int., 42 (2002), 717.

11) H. Lei and J. C. He: J. Non-Cryst. Solids, 352 (2006), 3772.

12) B. G. Thomas, L. J. Mika and F. M. Najjar: Metall. Trans. B, 21 (1990), 387.

13) H. Yang, L. Zhao, X. Zhang and K. Deng: Metall. Mater. Trans. B, 29 (1998), 1345

14) H. Lei, K. Nakajima and J. C. He: ISIJ Int., 50 (2010), 1735.

15) K. Asano and T. Nakano: Tetsu-to-Hagané, 57 (1971), 1943.

16) Q. Q. Lu, J. R. Fontaine and G. Aubertin: Int. J. Multiphas. Flow, 19 (1993), 347.

17) Q. Q. Lu, J. R. Fontaine and G. Aubertin: Aerosol Sci. Technol., 17 (1992), 169.

18) J. Z. Zhang: Fractal, Tsinghua University Publishers, Beijing, China, (1995), 176 .

19) P. J. O'Rourke: PhD thesis, Princeton University, (1981).

20) K. Higashitani, R. Ogawa, G. Hosokawa and Y. Matsuno: J. Chem. Eng. Jpn., 15 (1982), 299.

21) S. Taniguchi, A. Kikuchi, T. Ise and N. Shoji: ISIJ Int., 36 (1996), s117.

22) A. Kumar, S. K. Choudhary and S. K. Ajmani: ISIJ Int., 52 (2012), 2305.

23) H. Yin, H. Shibata, T. Emi and M. Suzuki: ISIJ Int., 37 (1997), 936.

24) T. Mizoguchi, Y. Ueshima, M. Sugiyama and K. Mizukami: ISIJ Int., 53 (2013), 639. 